\title{
Numerical analysis on fiber-nonlinearity compensation for 4ASK 100-km SSMF transmission by using electrical butterfly operation for received signal sidebands
}

\author{
Ryoto Nakagawa ${ }^{1}$, Yuya Takanashi ${ }^{1}$, and Moriya Nakamura ${ }^{1, \text { a) }}$ \\ ${ }^{1}$ School of Science and Technology, Meiji University, \\ 1-1-1 Higashimita, Tama-ku, Kawasaki, Kanagawa, 214-8571, Japan \\ a) m_naka@meiji.ac.jp
}

Abstract: We investigated a novel demodulation technology based on our proposed electrical butterfly operation scheme which compensates for optical nonlinear distortion of conventional single-sideband (SSB) signals. In the scheme, phase-conjugated twin signals are transmitted as lower sideband (LSB) and upper sideband (USB) components of a dual sideband (DSB) signal modulated by a simple Mach-Zehnder modulator (MZM). The performance was evaluated by numerical simulation of 4-ary amplitude shift keying (4ASK) signals transmitted over a 100-km standard single mode fiber (SSMF). The error vector magnitude (EVM) was improved by about $20 \%$ when the input power was $10 \mathrm{dBm}$, compared with a conventional SSB-4ASK signal.

Keywords: optical nonlinearity compensation, SSB modulation, phaseconjugated twin waves

Classification: Fiber-Optic Transmission for Communications

\section{References}

[1] K. Takano, Y. Naganuma, and K. Nakagawa, "Performance analysis of optical single sideband modulation based on Mach-Zehnder interferometers and its dispersive fiber transmission," IEICE Trans. Commun., vol. E88-B, no. 5, pp. 1994-2003, May 2005. DOI: 10.1093/ietcom/e88-b.5.1994

[2] K.I.A. Sampath, K. Takano, and M. Sato, "Self-phase modulation based signal distortions of optical SSB-SC signal with pilot carrier," Optoelectronics and Communications Conference (OECC2016), ThB1-3, July 2016.

[3] L. Zhang, Q. Zhang, T. Zuo, E. Zhou, G.N. Liu, and X. Xu, "C-band single wavelength $100-\mathrm{Gb} / \mathrm{s}$ IM-DD transmission over $80-\mathrm{km}$ SMF without CD compensation using SSB-DMT," Optical Fiber Communication Conference (OFC2015), Th4A.2, March 2015. DOI: 10.1364/ofc.2015.th4a.2

[4] M.S. Erkılınç, M.P. Thakur, S. Pachnicke, H. Griesser, J. Mitchell, B.C. Thomsen, P. Bayvel, and R.I. Killey, "Spectrally efficient WDM Nyquist pulseshaped subcarrier modulation using a dual-drive Mach-Zehnder modulator and direct detection," J. Lightw. Technol., vol. 34, no. 4, pp. 1158-1165, Feb. 2016. DOI: 10.1109/JLT.2015.2497343 
[5] X. Yi, M. Zhu, J. Zhang, X. Huang, and K. Qiu, "High-speed optical SSB transmissions using direct detection," Optoelectronics and Communications Conference (OECC2018), 4B4-1, July 2018. DOI: 10.1109/oecc.2018.8729890

[6] X. Liu, A.R. Chraplyvy, P.J. Winzer, R.W. Tkach, and S. Chandrasekhar, "Phase-conjugated twin waves for communication beyond the Kerr nonlinearity limit," Nature Photon., vol. 7, pp. 560-568, July 2013. DOI: 10.1038/ NPHOTON.2013.109

[7] T. Sakamoto, G.-W. Lu, and T. Kawanishi, "Oppositely-biased dual-polarization IQ modulators for nonlinearity-tolerant polarization-multiplexed phaseconjugated twin-signals generation," European Conference on Optical Communication (ECOC2015), Th.1.4.6, Sept. 2015. DOI: 10.1109/ecoc.2015.7341854

[8] H. Eliasson, P. Johannisson, M. Karlsson, and P.A. Andrekson, "Mitigation of nonlinearities using conjugate data repetition," Opt. Express, vol. 23, no. 3, pp. 2392-2402, Feb. 2015. DOI: 10.1364/OE.23.002392

[9] R. Nakagawa, Y. Takanashi, and M. Nakamura, "Phase-conjugated twin-SSB for compensation of optical nonlinear waveform distortion," Optoelectronics and Communications Conference (OECC2019), TuP4-B9, July 2019. DOI: 10.23919/PS.2019.8817812

[10] T. Sakamoto, G.-W. Lu, and T. Kawanishi, "Conjugated-paired radio-on-fiber transmission scheme highly tolerant against optical fiber nonlinearity," International Topical Meeting on Microwave Photonics (MWP2014), WD-6, Oct. 2014. DOI: $10.1109 / \mathrm{mwp} .2014 .6994583$

[11] Z. Xu, R. Hui, and M. O'Sullivan, "Dual-band OOFDM system based on tandem single-sideband modulation transmitter," OSA Opt. Express, vol. 17, no. 16, pp. 13479-13486, Aug. 2009. DOI: 10.1364/OE.17.013479

[12] L. Zhang, T. Zuo, Q. Zhang, J. Zhou, E. Zhou, and G.N. Liu, "Single wavelength 248-Gb/s transmission over 80-km SMF based on twin-SSB-DMT and direct detection," European Conference on Optical Communication (ECOC2016), M.2.D.2, Sept. 2016.

[13] M. Nakamura, S. Kashiwagi, Y. Takanashi, R. Nakagawa, and R. Nakamura, "Novel optical twin-SSB detection scheme using an electric butterfly operation," IEICE Commun. Express, vol. 8, no. 8, pp. 305-310, Aug. 2019. DOI: 10.1587/ comex.2019XBL0071

\section{Introduction}

Optical single sideband (SSB) modulation has been attracting attention due to its high spectral efficiency and dispersion-tolerant characteristics $[1,2,3,4,5]$. However, the high peak-to-average power ratio (PAPR) of an optical SSB signal induces waveform distortion caused by optical nonlinearities such as self-phase modulation (SPM) [2]. Consequently, SSB schemes require some nonlinear compensation to enable long-haul transmission [5]. A method using phase-conjugated twin waves is one of the important candidates for mitigating the optical nonlinear effects [6]. In this method, a phase-conjugated optical signal is polarization multiplexed or timedivision multiplexed with the original signal [7, 8]. In our study, we proposed a novel phase-conjugated twin scheme where the original optical SSB signal and the phase-conjugated SSB signal are multiplexed as a lower sideband (LSB) and upper sideband (USB) in the form of a twin-SSB optical signal [9]. A similar kind of phase-conjugated twin-signal method has been proposed by Sakamoto et al. [10]. 
In our proposed scheme, however, subcarrier modulation is not needed because the LSB and USB are completely demultiplexed by an electronic butterfly operation. In this paper, besides our former study in [9], we investigated the performance of the proposed scheme with varying input optical power to the optical fiber, and compared the performance with that of the conventional SSB scheme. The results show the optical-nonlinearity-tolerant characteristics of the proposed scheme.

\section{Principle of nonlinearity compensation of SSB signal}

Compared with a double sideband (DSB) signal, an SSB signal has dispersiontolerant characteristics due to the narrower bandwidth of the occupied spectrum. A twin-SSB signal conveys two independent signals as an LSB and a USB, as shown in Fig. 1(a). The twin-SSB scheme can achieve higher spectral efficiency because two different spectra can be arranged side by side without any spectral spacing $[11,12,13]$. Furthermore, the two independent signals on the LSB and USB are modulated using a single optical modulator, allowing a cost-effective transmitter to be realized. Figure 1(b) shows the structure of our proposed twin-SSB receiver consisting of homodyne detection and an electronic butterfly operation with a Hilbert transform [13]. By using this butterfly operation, the signals on the LSB and USB can be demultiplexed without using optical filters. The twin-SSB signal, $s(t)$, can be expressed as

$$
s(t)=\cos \left(\omega_{C}-\omega_{S L}\right) t+\cos \left(\omega_{C}+\omega_{S U}\right) t
$$

where $\omega_{S L}$ and $\omega_{S U}$ are the angular frequencies of the baseband signals for the LSB and USB, respectively, and $\omega_{C}$ is the optical carrier frequency. When homodyne detection is performed on this signal, the two outputs of the optical $90^{\circ}$-hybrid, $r_{1}(t)$ and $r_{2}(t)$, are expressed as

$$
\begin{aligned}
& r_{1}(t)=\frac{1}{2}\left\{\cos \left(\omega_{S L} t\right)+\cos \left(\omega_{S U} t\right)\right\}, \\
& r_{2}(t)=\frac{1}{2}\left\{\sin \left(\omega_{S L} t\right)-\sin \left(\omega_{S U} t\right)\right\},
\end{aligned}
$$

When a Hilbert transform is performed on $r_{2}(t)$, the result, $r_{3}(t)$, is expressed as

$$
r_{3}(t)=\frac{1}{2}\left\{-\cos \left(\omega_{S L} t\right)+\cos \left(\omega_{S U} t\right)\right\},
$$

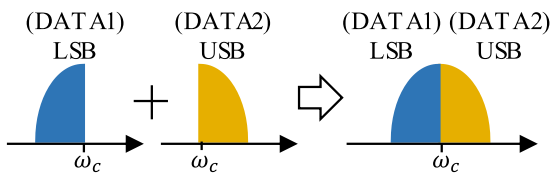

(a) Twin-SSB scheme.

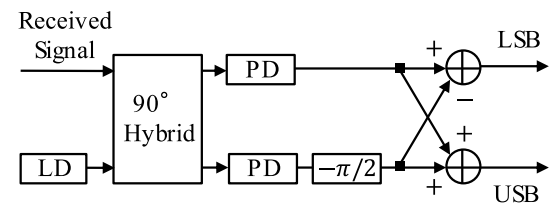

(b) Construction of twin-SSB receiver.

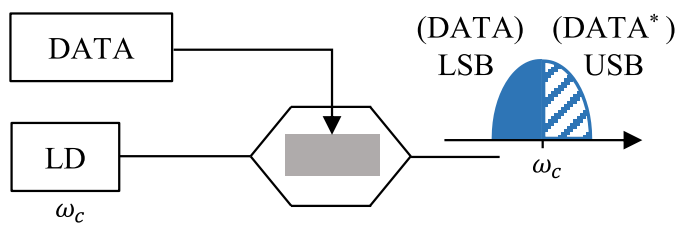

(c) Phase-conjugated relation of LSB and USB of DSB signal modulated by a single MZM.

Fig. 1. Principle of modulation and demodulation. 
By performing a butterfly operation on $r_{1}(t)$ and $r_{3}(t)$, the baseband signals of the LSB and USB can be retrieved as

$$
\begin{aligned}
& r_{1}(t)-r_{3}(t)=\cos \left(\omega_{S L} t\right), \\
& r_{1}(t)+r_{3}(t)=\cos \left(\omega_{S U} t\right) .
\end{aligned}
$$

In the proposed scheme, the original signal and the phase-conjugated signal are multiplexed and transmitted in the form of twin-SSB. It is known that this type of the modulated signal can be easily achieved by DSB intensity modulation using a single Mach-Zehnder modulator (MZM), as shown in Fig. 1(c) [10]. When the baseband signal and the optical carrier are expressed as $\cos \left(\omega_{s} t\right)$ and $\cos \left(\omega_{c} t\right)$, respectively, the modulated signal $s(t)$ can be expressed as

$$
\begin{aligned}
s(t) & =\frac{1}{2}\left\{\cos \left(\omega_{C}-\omega_{S}\right) t+\cos \left(\omega_{C}+\omega_{S}\right) t\right\} \\
& =\frac{1}{2} \operatorname{Re}\left\{\left(e^{-j \omega_{s} t}+e^{j \omega_{s} t}\right) \cdot e^{j \omega_{c} t}\right\},
\end{aligned}
$$

where $e^{-j \omega_{s} t}$ and $e^{j \omega_{s} t}$ are the complex amplitudes of the LSB and USB, respectively. The two complex amplitudes are in a phase-conjugate relationship. This signal on LSB and the phase-conjugated signal on USB are transmitted as a DSB. The multiplexed LSB and USB signals can be demultiplexed by the butterfly operation explained above. The nonlinear distortion can be compensated for by superimposing the LSB and USB components, because the nonlinear distortions of the phaseconjugated twin waves are anticorrelated [6].

\section{System setup}

The performance of the scheme was investigated by numerical simulations with a transmission system shown in Fig. 2. 10-Gsymbol/s 4-ary amplitude shift keying (4ASK) DSB modulation was performed using a single MZM. The single MZM was biased at the null point so that the optical carrier was suppressed. The LSB and USB components of the DSB signal were in a phase-conjugate relationship, as explained above. We used PRBS $2^{11}-1$ data. The modulated signal was transmitted by a $100-\mathrm{km}$ standard single mode fiber (SSMF). The loss parameter and dispersion coefficient were $0.2 \mathrm{~dB} / \mathrm{km}$ and $16.75 \mathrm{ps} / \mathrm{nm} / \mathrm{km}$, respectively. The input power to the optical fiber was adjusted from 0 to $10 \mathrm{dBm}$ using an optical attenuator (ATT). The noise figure of the erbium-doped fiber amplifiers (EDFAs) was $5 \mathrm{~dB}$. On the receiver side, we performed homodyne detection using a local oscillator (LO) with

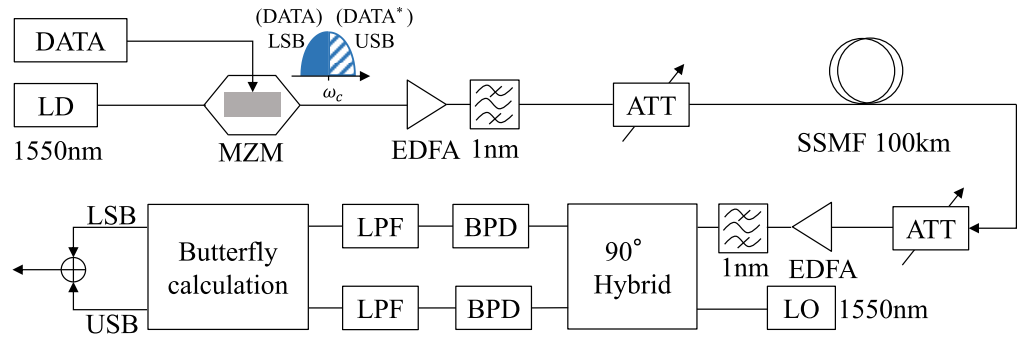

Fig. 2. System setup. 


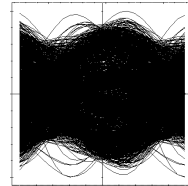

(a) SSB (LSB)

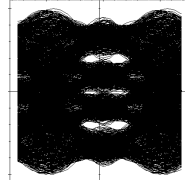

(b) DSB

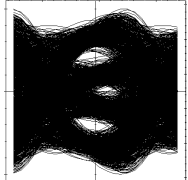

(c) LSB component

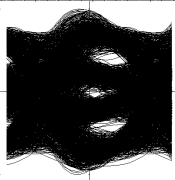

(d) USB component (e) LSB comp. +USB comp
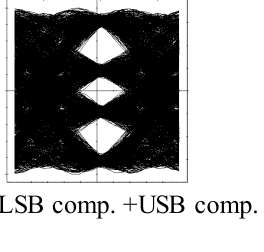

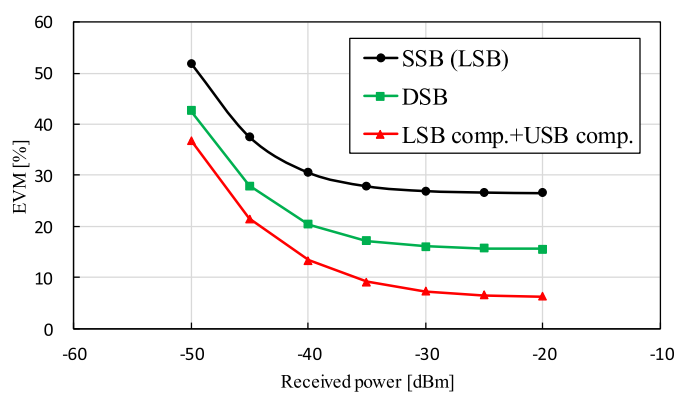

(f) EVM characteristics (Input power to optical fiber : $10 \mathrm{dBm}$ )

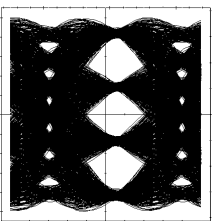

(g) Input power : $0 \mathrm{dBm}$

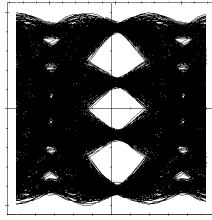

(h) Input power : $7 \mathrm{dBm}$

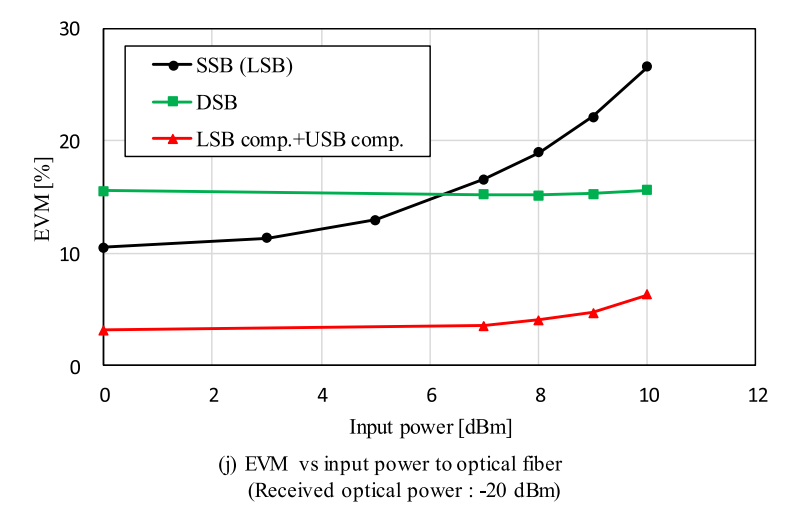

Fig. 3. Results of numerical simulations.

an optical power of $10 \mathrm{dBm}$. Here, we assumed that the $\mathrm{LO}$ was ideally synchronized to the optical signal. The thermal power density of balanced-photodiodes (BPDs) was $1.0 \times 10^{-22}[\mathrm{~W} / \mathrm{Hz}]$. The LSB and USB components were demultiplexed by the electronic butterfly operation. Nonlinear compensation was performed based on the scheme of phase-conjugated twin waves.

\section{Results and discussion}

Figures 3(a) to (e) show the eye diagrams of the received signal when the input power to the optical fiber was $10 \mathrm{dBm}$. The received optical power was $-20 \mathrm{dBm}$. Figure 3(a) shows the result of a standard SSB signal transmission demonstrated for comparison. The waveform of the LSB signal was completely distorted by SPM due to the large PAPR of the signal. Figure 3(b) shows the result of a normal homodyne detection of the DSB signal, and the waveform was distorted principally by chromatic dispersion. Figures 3(c) and (d) show the eye diagrams of the demultiplexed LSB and USB components of the DSB signal, respectively. Figure 3(e) shows the eye diagram 
after the nonlinear compensation. It was confirmed that eye opening was improved compared with the SSB signal due to the nonlinear compensation based on phaseconjugated twin waves. Figure 3(f) shows the error vector magnitude (EVM) versus the received optical power when the input optical power was $10 \mathrm{dBm}$. Here, we calculated EVM values at the optimum timings when the values became minimum. In the case of SSB, the EVM characteristics were more highly degraded by nonlinear effects compared to the DSB signal. By using the proposed scheme, however, the EVM was improved by about $20 \%$ compared to SSB and by about $9 \%$ compared to DSB. In the range of the lower received optical power, the EVM was degraded by the ASE noise of EDFAs and the thermal noise of BPDs. Figures 3(g) to 3(i) show the eye diagrams after the nonlinear compensation when the input optical power was changed from $0 \mathrm{dBm}$ to $10 \mathrm{dBm}$. Thanks to the optical nonlinearity tolerant characteristics of the proposed scheme, we did not observe a significant change of the eye opening. Figure $3(j)$ shows the EVM versus the input power to the optical fiber. When the input power was low enough, the EVM of the SSB signal was about 5\% better than DSB due to the dispersion tolerant characteristics. When the input power was large, however, the EVM of the SSB signal was seriously degraded by nonlinear effect. On the other hand, in the case of our proposed scheme, the EVM degradation caused by the large input power of $10 \mathrm{dBm}$ was small, at less than about $3 \%$. Additionally, even when the input power was low, we observed dispersion-tolerant characteristics. When the input power was $0 \mathrm{dBm}$, the EVM was improved by about $7 \%$ compared to conventional SSB. Finally, the mainlobe bandwidth of the 10-Gsymbol/s 4ASK DSB modulation was $20 \mathrm{GHz}$, giving a spectral efficiency of $1.0 \mathrm{bit} / \mathrm{s} / \mathrm{Hz}$. SSB modulation can double the spectral efficiency, achieving $2.0 \mathrm{bit} / \mathrm{s} / \mathrm{Hz}$. However, the spectral efficiency of the proposed scheme becomes $1.0 \mathrm{bit} / \mathrm{s} / \mathrm{Hz}$ as in the case of DSB.

\section{Conclusions}

We proposed a novel nonlinear compensation scheme for SSB signals. The scheme may be categorized as one type of phase-conjugated twin wave scheme. The modulation can be performed simply by using a single MZM. Demultiplexing is performed in the digital domain without using costly additional optical components. This simple construction of the proposed scheme is a major advantage over other methods. 